\title{
BMJ Open Understanding frailty: a qualitative study of European healthcare policy- makers' approaches to frailty screening and management
}

\author{
Holly Gwyther, ${ }^{1}$ Rachel Shaw, ${ }^{1}$ Eva-Amparo Jaime Dauden, ${ }^{2}$ Barbara D'Avanzo, ${ }^{3}$ \\ Donata Kurpas, ${ }^{4}$ Maria Bujnowska-Fedak, ${ }^{4}$ Tomasz Kujawa, ${ }^{4}$ Maura Marcucci, ${ }^{5}$ \\ Antonio Cano, ${ }^{2}$ Carol Holland ${ }^{6}$
}

To cite: Gwyther $\mathrm{H}$, Shaw $\mathrm{R}$, Jaime Dauden E-A, et al. Understanding frailty: a qualitative study of European healthcare policy-makers' approaches to frailty screening and management. BMJ Open 2018;8:e018653. doi:10.1136/ bmjopen-2017-018653

- Prepublication history and additional material for this paper are available online. To view these files, please visit the journal online (http://dx.doi org/10.1136/bmjopen-2017018653).

Received 14 July 2017 Revised 26 September 2017 Accepted 4 October 2017

CrossMark

For numbered affiliations see end of article.

Correspondence to

Dr Holly Gwyther;

h.gwyther@aston.ac.uk

\section{ABSTRACT}

Objective To elicit European healthcare policymakers' views, understanding and attitudes about the implementation of frailty screening and management strategies and responses to stakeholders' views.

Design Thematic analysis of semistructured qualitative interviews.

Setting European healthcare policy departments. Participants Seven European healthcare policy-makers representing the European Union ( $n=2)$, UK $(n=2)$, Italy $(n=1)$, Spain $(n=1)$ and Poland $(n=1)$. Participants were sourced through professional networks and the European Commission Authentication Service website and were required to be in an active healthcare policy or decisionmaking role.

Results Seven themes were identified. Our findings reveal a 'knowledge gap', around frailty and awareness of the malleability of frailty, which has resulted in restricted ownership of frailty by specialists. Policy-makers emphasised the need to recognise frailty as a clinical syndrome but stressed that it should be managed via an integrated and interdisciplinary response to chronicity and ageing. That is, through social co-production. This would require a culture shift in care with redeployment of existing resources to deliver frailty management and intervention services. Policy-makers proposed barriers to a culture shift, indicating a need to be innovative with solutions to empower older adults to optimise their health and wellbeing, while still fully engaging in the social environment. The cultural acceptance of an integrated care system theme described the complexities of institutional change management, as well as cultural issues relating to working democratically, while in signposting adult care, the need for a personal navigator to help older adults to access appropriate services was proposed. Policy-makers also believed that screening for frailty could be an effective tool for frailty management.

Conclusions There is potential for frailty to be managed in a more integrated and person-centred manner, overcoming the challenges associated with niche ownership within the healthcare system. There is also a need to raise its profile and develop a common understanding of its malleability among stakeholders, as well as consistency in how and when it is measured.
Strengths and limitations of this study

- To the best of our knowledge, this is the first qualitative study with European healthcare policymakers to focus on the implementation of frailty screening and management strategies.

- Use of semistructured interviews allowed us to collect detailed insights into policy-makers' views, understanding and attitudes towards frailty management, screening and prevention programmes.

- The main limitation is that the sample size is unavoidably small. There are only a few policymakers in senior healthcare positions within the European Commission or at a senior level nationally, and thus, as elites, the potential pool of participants is itself very small.

\section{INTRODUCTION}

Frailty can be conceptualised as a multidimensional, clinical condition related to age, during which multiple physiological and psychological systems gradually lose their reserves, and individuals become less able to cope with daily stressors or acute illnesses. Older adults living with frailty are more vulnerable to adverse health outcomes, including institutionalisation and mortality, particularly when exposed to events such as a chronic disease diagnosis, an acute infection or a fall. ${ }^{1-3}$

Research suggests that frailty is a dynamic process $^{4}$ and that there are opportunities along its pathway to transition out of, manage and/or prevent its adverse consequences. ${ }^{5-11}$ Early identification of frailty through screening programmes may provide the opportunity to identify prefrail and frail individuals, and direct them to appropriate preventative health interventions to assist them to improve personal health and 
well-being, resulting in better management of societal healthcare costs. ${ }^{12} 13$

Operational concepts of frailty have moved on from the earlier physiological phenotype ${ }^{14} 15$ or accumulation of deficits models. ${ }^{16}{ }^{17}$ A broader multidimensional approach for measuring frailty ${ }^{19}$ has been adopted that also acknowledges psychological elements like wellbeing and quality of life, and social elements such as lack of social contacts or environmental and situational factors. ${ }^{19-21}$ Embracing this approach, some studies have tested and noted success with multicomponent interventions. In varying combinations, interventions incorporating physical training, cognitive training, nutritional advice and social support have resulted in significant improvements in frailty measures in community dwelling older adults ${ }^{822}$ and people in residential care homes. ${ }^{23}$ (For reviews, see references 24 and $25^{24}{ }^{25}$ ). Such multicomponent interventions may also prevent future health risk and social isolation.

Against this background of evidence for multicomponent interventions, this study forms the second phase of qualitative research with stakeholders on frailty prevention and screening. The first phase ${ }^{26}$ aimed to explore how frailty prevention and screening would be accepted and adopted by European stakeholders, including frail and non-frail older adults, family caregivers, and health and social care professionals. Previously, older adults' and other stakeholders' views on frailty screening had not been sought. The findings from the first phase ${ }^{26}$ demonstrated consistent results across the three countries involved (UK, Poland and Italy), and emphasised the need for a holistic approach to frailty care and early intervention. Participants raised the need for integrated and coordinated health and social care services, as well as personalised screening programmes and advocacy in the organisation of care. Central to all stakeholders was the significance and primacy of the psychological and social elements of frailty. Physical frailty was thought of as less malleable or preventable, and of less importance provided individuals retained psychological resilience. Furthermore a meta-synthesis of qualitative evidence ${ }^{25}$ highlighted the need to understand the acceptability of frailty screening among the general population of older adults, their caregivers and other stakeholders, including the health and social care staff who may conduct assessments or deliver interventions, and to address the understanding of the malleability, reversibility or preventability of frailty.

This study completes the picture by exploring European healthcare policy-makers' opinions on frailty and the feasibility of frailty screening programmes and healthcare interventions suggested by stakeholders during this earlier work, examining their responses to the findings of the first phase. ${ }^{25} 26$

\section{METHOD}

This study is part of a larger programme, 'Frailty Management Optimisation through EIP-AHA Commitments and
Utilisation of Stakeholders Input' (FOCUS), funded by the EU (Grant number 664367 FOCUS) (http://focus-aha.eu/ en/home) ${ }^{27}$ The methods used in this study conform to qualitative research reporting guidelines. ${ }^{28}$

\section{Participants}

Healthcare policy-makers working at regional, national and European levels were purposively sampled. Participants were sourced through professional networks and the European Commission Authentication Service. They were required to be in an active healthcare policy and/or decision-making role and have experience of frailty policy or frailty related healthcare policy. Two participants did not respond to a request for interview, no reason was provided. Volunteers were given participant information sheets in English and understanding checked where interviews were conducted in a local language. Informed consent was received. In order to retain confidentiality, contributors' names, job titles, job descriptions, precise geographical locations and service names were anonymised (see table 1).

\section{Data collection}

Individual interviews were conducted in English (except in Poland and Spain) and lasted between $30 \mathrm{~min}$ and $90 \mathrm{~min}$. Semistructured interviews were conducted in person (UK and Poland), over the telephone (UK, Luxembourg, Belgium and Italy) or by video conference (Spain). Only interviewers and participants were present. The interview schedule was defined in advance based on previous findings from stakeholder focus groups.$^{26}$ Before the interview, all participants were sent a summary of the findings of the previous study (see online supplementary appendix $1)$. The most prominent themes from the previous findings were distilled into seven questions, with subordinate questions, to stimulate conversation (see online supplementary appendix 2). The same question list was used by all interviewers but within that, slightly different questions were asked of each policy-maker, dependent on their area of expertise. Questions were not pilot tested. At interview, we (A) made clear the stakeholders' opinions and needs to policy-makers; (B) asked them about the feasibility of the implementation of the needs and suggestions that had emerged during stakeholder discussion and $(\mathrm{C})$ collected policy-makers' possible proposals to better address stakeholders' concerns.

Interviews were facilitated by female researcherspsychologists (BD and HG) based in Italy and the UK, respectively, and a general practitioner (DK) in Poland. The interview in Spain was conducted by a male senior professor of obstetrics and gynaecology with a female project manager (AC and E-AJD). All interviewers had previous experience in qualitative research. No previous relationships existed between interviewers and participants and no personal information was relayed about the researchers to the participants except in Italy, where the interviewer was known to the policy-maker. Given the status of participants in each country, their contributions 


\begin{tabular}{|c|c|c|c|}
\hline Country & Title & Organisation & Role description \\
\hline UK & Clinical Director & Secondary healthcare & $\begin{array}{l}\text { Consultant physician with experience of unification of healthcare } \\
\text { treatment protocols, medical interventions and sharing good } \\
\text { practices. }\end{array}$ \\
\hline Belgium & Head of Unit & $\begin{array}{l}\text { European Commission } \\
\text { (Directorate-General } \\
\text { (DG) Santé) }\end{array}$ & $\begin{array}{l}\text { Policy role in EU with experience of health strategy development } \\
\text { and analysis. }\end{array}$ \\
\hline UK & $\begin{array}{l}\text { Clinical Lead for } \\
\text { Integrated Care }\end{array}$ & National Government & $\begin{array}{l}\text { Consultant physician with experience of policy development in } \\
\text { integrated care and reshaping healthcare for older adults. }\end{array}$ \\
\hline Luxembourg & $\begin{array}{l}\text { Programme } \\
\text { Coordinator }\end{array}$ & $\begin{array}{l}\text { European Commission } \\
\text { (DG Santé) }\end{array}$ & $\begin{array}{l}\text { Policy role in public health with experience of implementing health } \\
\text { programmes. }\end{array}$ \\
\hline Italy & Managing Director & $\begin{array}{l}\text { Directorate General } \\
\text { Welfare }\end{array}$ & Policy and service planning with experience at a regional level. \\
\hline Spain & Deputy Director & Regional Government & Physician. Policy role at the Public Health Directorate. \\
\hline
\end{tabular}

represent a rich, contextualised understanding of healthcare policy perspectives on frailty and frailty management across Europe.

\section{Data analysis}

Discussions were digitally audio recorded and transcribed verbatim in the language of origin. Translations from the original language to English were reviewed by a native English speaker for syntactical structure and conceptual equivalence. Minor amendments were made to literal translations to ensure that participants' words were accessible and understandable. Amendments were checked with the translator to ensure that the original meaning had not been lost. Transcriptions were analysed by HG and RS using thematic analysis ${ }^{29}$ with coding generated both inductively and deductively. The process of inductive coding involved thorough and repeated reading of transcripts to develop a set of preliminary themes. Notes were made independently by analysts to highlight issues raised by policy-makers, and a search for patterns was conducted. Codes were assigned, collated and compared within and across transcripts. Themes were then developed through discussion and further independent interpretative work until consensus was reached within the wider team. Codes were also devised deductively from questions asked during the interview process and grouped into themes. Finally, concepts were clustered and synthesised into the interpretation presented here. Validity was protected by the use of transparent procedures and through constant exchange between interviewers (HG, BD, E-AJD, DK, TK) and analysts (HG, RS). A summary of findings, including verbatim data extracts was circulated to participants for comments. Six of the seven participants responded. They verified findings and stated that it captured the range of viewpoints successfully. Only minor amendments were made.

\section{Patient involvement}

No patients were directly involved in this research. However, the views of previous focus group participants including frail and non-frail older adults were used to generate the interview schedule. ${ }^{26}$

\section{RESULTS}

\section{Sample characteristics}

Participants' characteristics are reported in table 1. Limited information is provided to protect anonymity and retain confidentiality.

To illuminate study findings, each theme is presented with example quotations. Quotations are attributed to each policy-maker using a unique participant code.

\section{Awareness of the malleability of frailty}

Policy-makers' initial perception was that, despite increasing exposure to frail older adults, there was a lack of awareness around frailty; a 'knowledge gap' (PM01) among general clinicians and allied health professionals. Policy-makers raised the idea that frailty was not being effectively managed in the current acute care system and that while this could potentially be attributed to a variety of factors, a fundamental issue was a lack of understanding of the nature of frailty, and in particular its malleability.

most of the professionals who work with adults and older people will be coming across frailty every day. They might not recognise what they can do about it but I think that they're aware of it as a challenge. I don't think they're fully aware of what the possibilities are. (PM03)

A common thread throughout this and other policy-makers' accounts was the growing challenge of the changing population demographics and the increasing numbers of health professionals who encounter frailty. Policy-makers described a pressing need to raise the 
profile of frailty and consequently facilitate more effective management of frailty and frailty related conditions.

I think fundamentally the key thing is that there should be a campaign about frailty, so that people start hearing that word more often and then they understand what frailty means. Simultaneously we need to have a dialogue with all the healthcare providers and key commissioners [...] At the healthcare professional level we need to create awareness, we need to train them to address frailty and also try to provide resources. (PM01)

Furthermore, there were suggestions that clinical personnel should be better trained in recognising and managing frailty, specifically physicians, allied health professionals and healthcare policy-makers including healthcare commissioners. The view here was that without raising the profile of frailty at all levels within the healthcare system and 'broadening the debate' (PM02), little of significance could be achieved, in terms of allocating resources to frailty management and care. This was clarified by one policy-maker:

Currently, the awareness around frailty is poor, then whatever we talk about afterwards is not going to happen, until we address this primary deficiency. (PM01)

Although some policy-makers demonstrated an understanding of the malleability of frailty, there were others who implied that frailty was a normal part of ageing and as such was not entirely preventable or reversible.

There will be always frail people, but if we know what are the triggers for becoming frail, what are the diseases which then end up as, as a chronic disease which have frailty as a consequence. There, if you would know all this, you know, you should be acting in a very early stage for people to not get into the frailty stage, so in this sense, yes of course [frailty is preventable] but $100 \%$ [preventable], no. (PM04)

Despite some doubt about the malleability, reversibility and absolute preventability of frailty, which we acknowledge may be unachievable, the above extract demonstrates a strong conviction towards understanding the mechanisms of frailty in order to treat patients effectively.

\section{Ownership of frailty}

Policy-makers' described that frailty management was currently owned by specialists.

I think to a large extent the debate on frailty has been part of the geriatricians and some gerontologists also, so it's kind of a speciality issue. (PM02)

They suggested that ownership of frailty should be devolved from these specialists to a wider healthcare audience through an awareness raising campaign and training programme. For some, this meant categorising frailty as a clinical syndrome requiring intervention, like any other. Certainly, this would enable transparency in the management of this chronic condition and empower health professionals to extend their role in patient care. However, these ideas were limited, in that they were only associated with raising awareness and introducing expertise within the healthcare system. Other policy-makers, specifically those involved in reforming or integrating their respective health and social care systems, took these views one stage further. They advocated that ownership should be extended to the wider community:

I think that for decades or so, frailty has been very bio-medical and I think the real potential to unlock the opportunities is if we move out of that domain and see it as an area where there is huge potential from community capacity building, to community led interventions, social connectedness, that I think then brings it to a level of potential reversibility. (PM03)

And the health system ... has to become more open, more inclusive, less deified, and it has to realise that the solution is not only on the 'white coat' but this is a shared solution and there has to be a co-leadership or social co-production. (PM07)

Superficially, there appeared to be a dichotomy in beliefs about frailty management. On one hand, some policy-makers appeared to support a greater medicalisation of frailty, a need for frailty to be recognised as an authentic clinical issue by medical professionals and treated as such. On the other, there were views that frailty should be demedicalised and that frailty management should be conceived of as an adaptation to life stages and be embraced as a societal issue with ownership devolved to a wider societal network. On further examination, it was determined that these views were not mutually exclusive but rather described a spectrum of ownership of frailty, representing different degrees of enablement and empowerment for frail older adults.

Conducting training and developing knowledge in healthcare professionals ensures that frail older adults are treated with compassion and dignity, and crucially, enables them to find support to make informed choices about their own health and healthcare. Certainly, several policy-makers endorsed the view that patient input and empowerment was important within care systems.

I think that the issue here is that we should listen more to the affected people and we must develop more protocols, guidelines, programmes with the affected people and not exclusively from the expert, professional or public health opinion. (PM07)

Ownership has implications for both costs and treatment. Given ongoing changes in population demographics, reducing the burden on the healthcare system by involving the wider community provides a financially sustainable solution. Also, given the multicomponent, biopsychosocial nature of frailty, a purely medical/physical approach to frailty may fail to address some or all of 
the cognitive and/or social components associated with the condition.

In summary, this theme challenges the role of the hospital and specialists as the dominant force in frailty care. It provides an opportunity for medical professionals, specifically specialists, to share ownership of frailty, certainly with other healthcare professionals in the short term and also to actively engage with the wider community. In doing this, a culture shift in care could be facilitated and older adults empowered to take greater control of their later life journey, which will be discussed next.

\section{A culture shift in care}

Participants judged that there was no simple solution to the frailty challenge and that there was no single body responsible for leading a culture change.

It's all stakeholders. There's no one person that owns this. There's no one sector that owns it. There's no one group of professionals that owns it so it's got to be across public sector, a cross-government kind of approach. (PM03)

Shifts in ownership of frailty would constitute a significant shift in the prevalent models of care. Policy-makers were aware that the current model of acute care was not effective in managing complex, chronic conditions such as frailty and that a culture shift was required to adapt to the changing needs of the population.

I think that it is increasingly consensual that our current health care models are not so conducive to looking at this sort of complex cases. I mean you have a lot of, increasingly you hear about, issues about multimorbidity for instance but still our healthcare systems are geared to single chronic diseases. (PM02)

There was a recognition that frailty and frailty management are complex issues involving many stakeholders and numerous components. This was a view supported by other policy-makers who described frailty as a 'puzzle" (PM01). Participants noted the multiplicity of stakeholders involved in the creation of a 'new' culture and suggested that in order to facilitate change, a multilateral approach to raising awareness, from the 'bottom up' (general public) and the 'top down' (healthcare commissioners) would be necessary.

In terms of delivery of a more appropriate system, policy-makers described a model of integrated and person-centred care in which frail older adults are treated as a whole, rather than as a fragmented collection of illnesses. They also described a system that would empower older adults to reduce their dependency on others, and ultimately conserve resources.

If somebody needs assistance in washing and dressing we tend to enable them, whatever their daily needs but we are not enabling them to make themselves more capable of doing that. I think if we were addressing frailty it would get us to that point and in fact, it might save, eventually, in the long term, some of the costs associated with caring because at least, even a small proportion get self-caring, is still beneficial. (PM01)

There was also a warning by one policy-maker that medicalising frailty and singling it out as a clinical syndrome would disrupt recent progress in care integration.

I think that one of the biggest challenges and the biggest risks is that if you put this into a frailty box. I think that this has got to be done in the context of this is about older people, this is about later life, this is about people centred integrated care and support. I think if we try to make it something that is different from what we've been talking about for the last ten, fifteen years around chronic disease, we'll fail. (PM03)

Supporting this and developing it further was another policy-maker's 'salutogenic' (PM07) perspective addressing cultural norms about health and the way in which contemporary society focuses on the absence of health or the presence of an acute/chronic condition. Within this idea, they described how an experience of frailty could potentially be embraced as a way of opening up a range of possibilities, interactions and opportunities, particularly within the community, 'a social prescription' (PM07). In their thoughts about care integration, they spoke about a concept of citizenship, a social movement, 'carezenship' as a marker of an advanced society, an advanced citizenship, one in which citizens take care of themselves (both on a personal and societal level) and learn to value care. However, they acknowledged that this would be a challenge.

We are asking a lot of ourselves to reorient programmes and interventions with a salutogenic approach, and give prominence to people, to the people themselves, rather than the scope of needs that generate demand for services, and professionals to look after needs, and also to create health based on assets, on these resources we all have. (PM07)

\section{Barriers to a culture shift}

Policy-makers described an integrated model of care as appealing in theory. They provided some evidentiary support, notably from pilot schemes, but they acknowledged that it would take time and thought to integrate into existing systems.

It's a bit of a long game and I think progress that has been made is now becoming even more challenging with the current fiscal environment. So, yes, we know it's the application of the chronic care model for long term conditions but doing that through a functional, a people-centred, integrated and functional lens, absolutely we know what we need to do, we just need to do it. (PM03) 
This latter extract raises potential barriers to an integrated and truly democratic healthcare system: there is a time element, an acknowledgement that this is a 'long game' (PM03) and that change management is a lengthy process; there is a need to be mindful of finances and resourcing issues; there is a need to take action, to implement the system or actually ' $d o$ it' (PM03); and there is a need to focus on patients' autonomy.

Despite acknowledging the difficulties and lack of funding for new projects in the current environment of austerity, policy-makers indicated that finances and resources were available and could potentially be reallocated to support a different system.

So, can this still be deliverable? The answer is yes but it needs a great deal of commitment, a great deal of fresh thinking to see how we are currently utilising our resources and see how we can change it to fit into this pathway and ... we've already got nutritionists, we've already got physiotherapists, we've already got occupational therapists and physical activity experts, but currently the way we utilise them is different and what we need to do is see whether there could be changes in the way they actually deliver their service with frailty as an underlying theme. (PM01)

This extract emphasises the need to do things differently, to think creatively and to 'rewire and use [assets] differently' (PM03), in order to better manage complex and chronic care needs. There is a sense that change is both possible and necessary, and that resources are available to facilitate this:

Since 2014 I would say we have had every year sizeable envelopes from different programmes from the [commissioner]. So the finance is available... ...so there has consistently been money for this even when it's not called, that it's not for frailty. (PM02)

Another challenge for resourcing is the fact that frailty is often subsumed within other remits and is viewed within a subset of many other chronic diseases of older adults. In fact, there was a strong sense from policy-makers that frailty could not be viewed in isolation or 'disconnected from the wider conversation about managing chronicity and complexity in care systems' (PM03) and therefore should not be funded separately. It is important to note though, that policy-makers state that a focus on frailty is required.

\section{Cultural acceptance of an integrated care system}

The complexities surrounding change management in large systems and institutions, as well as issues of cultural acceptance of a new way of working were described.

I think this [acute healthcare model] is a problem, not just in [region name] but all over the world...I think it will be a very slow process...I expect this process will take probably five to ten years before the culture of professionals will be ready because our doctors, our General Practitioners, our specialists and also social care workers are not trained to change their way. (PM05)

Indeed, the power transfer from senior clinicians to a wider range of potentially less well qualified staff in a truly democratic care pathway, implied in this extract, was an issue raised by a number of policy-makers. Policy-makers noted that senior clinicians may be reluctant to involve less well qualified people in decision-making processes.

What is integrated care? What is person centred care? What is joint decision making? ...it would, in several systems probably need a radical, a new thinking of... not this, 'I'm the doctor, I know what's good for you, this is what you're going to do' but a change in the doctor-patient relationship and also in the relationship among the different health personnel. You know, if suddenly the social carer has the same say in a discussion around a specific patient as the medical doctor. (PM04)

Policy-makers described cultural issues of medical hierarchy and physician dominance in healthcare, where power is exercised through the professional autonomy of doctors. There are issues here about the ability of doctors to treat allied health professionals and other stakeholders, for example carers, as equal counterparts in the care of frail adults. Further, there are issues of trust and reluctance on the part of the doctors to transfer power, knowledge and ownership of frailty management in a more democratic system; equally there may be reluctance on the part of other stakeholders to accept those responsibilities; thus resulting in fractures in care provision. Notwithstanding these cultural challenges, policy-makers were optimistic and believed that barriers could be overcome given sufficient time and training.

Several policy-makers were keen that any new frailty management system should be woven into a wider network of healthy ageing issues and delivered as a programme styled as 'living well for longer'. (PM03). The aim of this was to standardise approaches to multimorbidity and chronic disease, that is, to have the same pathway for all, and to overcome the negative connotations and language associations with frailty.

I think we're probably stuck with a fairly negative connotation from frailty, sounds as if it's something that means people are helpless, nothing you can do, whereas if we slip language and put the focus on ageing well, active and healthy ageing, living you know, living more positive fulfilling lives, then people could hang on to that, what's not to like about that? (PM03)

\section{Signposting adult care}

Stakeholders in the first phase described difficulties accessing care and navigating overly complex care systems. To overcome this, they expressed the need for an official 'wellbeing coordinator'. This suggestion for a new health 
visitor style role, as an advocate, monitor and source of information, was not supported by policy-makers.

Some policy-makers suggested that the role was unnecessary as the family doctor or General Practitioner (GP) could act as a care navigator.

I would like to think that a good General Practitioner, who has good understanding of frailty, can be themselves the coordinator, we don't need a separate person. (PM01)

Others suggested that GPs are already overburdened and that there are potentially more suitable people to take on this task.

Actually, it doesn't need to be the GP, the GP might not be the best placed person for that individual. It might be the mental health nurse, it might be the social worker, it might be the OT [occupational therapist], it might be [a] community connector. (PM03)

Policy-makers also raised the idea that new services, such as a well-being coordinator should be elective rather than prescriptive, which might also assist in controlling care costs.

So, I think the one size fits all is definitely not the way to go and personalised care, where the patient only receives the treatment that she really needs, maybe in the end this would actually be cost effective for the health system if you don't get everything just because a protocol foresees it. (PM04)

To summarise, the proposed new role of the wellbeing coordinator was not supported. However the policy-makers acknowledged that an enabled person in an existing role, a personal navigator is required to help older adults and their caregivers to access appropriate services.

\section{Screening for and preventing frailty}

Policy-makers were receptive to the idea of screening for frailty so long as it initiated a proactive consultative programme of care and interventions, a view which resonated with focus group members in the first phase of research. One policy-maker (PM07) made the point that screening was simply another method of 'medicalising' frailty and categorising a life stage. Despite doubts about the preventability and reversibility of frailty, there was a strong belief in the power of screening as a tool for effectively targeting those most in need of healthcare services and interventions, and directing resources accordingly.

If you are able to detect this health problem early and to know that you have the right measures to apply them and that they will really make improvements in life expectancy, in quality of life, then the benefit is really there. (PM07).

One participant raised concerns about the viability of frailty prevention interventions in an older, chronically ill population, that is, whether interventions are cost-effective and beneficial.
Screening is necessary. Is it viable? Most likely for the system, on balance, yes. Why? The problem of intervention in the case of these people, if we look to the future, must be focused on their specific health needs. So it is good to know which patients they are so we do not undertake unnecessary medical interventions. If a patient comes to a specialist who does not recognise frailty, they will want to cure the patient. So the question is, if frailty cannot be cured, are those interventions necessary from a medical standpoint? (PM06)

The perception that frailty is untreatable is also of interest, although this was not shared by all participants. Irrespective, there was an overwhelmingly positive view from policy-makers that screening followed by interventions are worthwhile:

I would really only support screening for frailty if it was linked with the kind of interventions that can make a difference. Otherwise, why are we doing the screening programme? It's about human values as much as pounds. (PM03)

Policy-makers were also concerned with wider issues, the practicalities of screening and the complexity of screening tools.

Any tool that is complex or that has multiple steps or requires quite a lot of in depth assessment might be quite difficult to perform as a screening tool, purely because the numbers we would be dealing with are going to be huge. We wouldn't have the resource (PM01)

There was a general consensus that a 'simple and easily reproducible' (PM01) or 'straightforward' (PM02) tool was required to minimise the resource and labour implications for health services and key professionals.

The affordability of population screening programmes was not a primary concern. Policy-makers agreed that there were ways of reducing screening costs through targeting strategies such as algorithms and e-health or self-assessment systems. Of more concern were the matters of when screening would occur and who would undertake it. One policy-maker had a clear opinion that it should not be undertaken by general practitioners.

and, who would be screening for frailty? You know, the GP? I don't know, is that another thing you want to burden on GPs? Did you speak to GPs about that? You know, we are putting so much on them, you know literally everything is on the GP in this regard, everything. (PM04)

Policy-makers were mindful of screening at 'key transition points' (PM03) in people's lives: including instances of acute illness, prescription review, bereavement and moving home/care setting. The view here was that screening and intervening at these critical points can significantly affect the incidence of adverse outcomes 
such as institutionalisation and dependency. Reverting back to the idea of an integrated and personalised system of care, there was also an understanding that an individual-specific 'care navigator' (PM03) would be present at the time of screening.

\section{DISCUSSION}

The data gathered have enabled us to consider which issues need to be addressed to assist in frailty management, screening and prevention programmes. We identified a knowledge gap regarding frailty and the need to raise awareness of frailty and the treatability of frailty throughout the medical profession through improved training and research. Healthcare professionals including the primary healthcare team, require an awareness of frailty and its treatability, as well as guidelines for best clinical practice, an overview of insights from new interventions and practical guidance on how to assess and manage frail individuals. Simultaneously, there is a need to reconsider the current system of "opportunistic' healthcare professional training on frailty, which may vary dependent on students' clinical placements, to ensure that all new healthcare practitioners develop a full understanding of the experiences of frail older adults. We also identified the need for frailty to be recognised as a clinical syndrome but managed within a broader remit of healthy ageing in the community.

Despite evidence to the contrary, our analysis found that some policy-makers, as well as other stakeholders, believe that frailty is not preventable or amenable to intervention. Since the effectiveness of any frailty management or screening programme relies on the prioritisation and allocation of resources and labour, this may lead to inequality in service provision across different jurisdictions. Further, there is a danger that screening programmes in these areas will classify older adults as frail or prefrail, identifying those who would benefit from early intervention but then fail to implement appropriate preventative treatments.

Policy-makers recognised the difficulties associated with managing frailty and the need to apply creative solutions to better organise and re-deploy existing services, resources, skills and knowledge sets to manage complex and chronic care needs.

We noted that policy-makers placed value on the development of an integrated and person-centred system of care, involving a care navigator, and while this idea is to some extent in its infancy, there was a strong sense that this was the preferred route. Certainly this system is in line with the chronic care model ${ }^{3031}$ which has been shown to improve patient care and result in improved outcomes in chronic conditions. ${ }^{32}$ Indeed, in some countries, this is beginning to happen. For example, in the UK, recent National Institute for Health and Care Excellence guidance ${ }^{33}$ for healthcare professionals, on the clinical assessment and management of multiple long-term conditions, recommended that a person's goals, values and priorities are established when determining care plans for adults with multimorbidity.
We confirmed the views of previous stakeholders ${ }^{26}$ that screening for frailty must have an outcome or specific purpose, and that outcomes are absolutely essential from a human and moral perspective. We have also identified that there must be an individually negotiated, person-centred and transparent care pathway available after screening for all frail older adults and that this pathway must be sufficiently flexible to adapt to individuals' needs, whether those are physical, cognitive or social. Underlying this culture shift in older adult care is the need to empower people to make informed choices about their own health and healthcare, but such empowerment requires high levels of perceived behavioural control ${ }^{34}$ and self-efficacy (self confidence in one's own ability to achieve a particular task, eg, ${ }^{35}$ ). In this case, believing that frailty is preventable and malleable provides the basis for that self-efficacy; the self-confidence to take charge of one's frailty prevention pathway is dependent on the belief that it is possible. ${ }^{34}$ Furthermore, self-efficacy requires ownership: older adults must take ownership of their frailty prevention pathway to engage in it as an active agent. This self-advocacy in turn depends on healthcare providers who are genuinely person-centred and able to enter into the true spirit of concordance, that is, a negotiated consultation which facilitates informed and collaborative decision-making. ${ }^{37}$ To achieve this, a psychological behaviour change intervention aimed at healthcare providers may be useful, to shift their approach from advice giving, which comes naturally to them and has been rehearsed for many years, towards a collaborative consultation which fosters authentic patient empowerment ${ }^{38}$ and takes older adults' freedom of choice seriously. From there, person-centred care, concordance, and the phenomenon of 'carezenship' (PM07) become a conceivable possibility, although a possibility that will require long-term focus and systemic investment to achieve.

\section{Strengths and limitations of the study}

To our knowledge, this is the first qualitative study with policy-makers from six European countries on frailty. There were some differences in the professional roles of the participants, due to the nature of the policy organisations in their respective countries and their professional background, for example, some were clinically qualified while others were civil servants, although all had significant experience of working in frailty related roles. Nevertheless, it was possible to distil information and compare across accounts, which demonstrated a great number of similarities, irrespective of background and/or role. Although the sample was relatively small, it was adequate to deliver the objective of the study and obtain valuable insights into policy-makers' perspectives. To further justify our sample size, there are only a few policy-makers in senior healthcare positions within the European Commission and at a senior level nationally within the respective countries and thus, as 'elites', the potential pool of participants is itself very small. To our knowledge, healthcare research with policy-makers at this level is limited. However, authors have described studies with fewer than 10 'ministry' level participants. 3940 
Further, in terms of data saturation, a sample size of six to twelve in a homogenous population has been described as 'sufficient to enable development of [high level] meaningful themes' (Guest $\mathrm{p} 78^{41}$ ) while others suggested that expertise in a topic can reduce the number of participants required in a study. ${ }^{42}$ Although data saturation is difficult to define ${ }^{43}$ it has come to be associated with the point at which no new information or themes can be gleaned from the data. ${ }^{41}$ While we cannot be absolutely sure that no new information would be discovered with additional interviews, the degree of commonality in responses enables us to fully answer our research questions and view our data as 'rich, full and complete'. (Morse p149 ${ }^{44}$ ).

\section{CONCLUSIONS AND IMPLICATIONS FOR CLINICIANS AND POLICY-MAKERS}

Frailty is a syndrome which crosses traditional medical discipline-specific boundaries. Our findings recommend a multilateral campaign of raising awareness of the malleability and preventability of frailty which targets health and social care professionals, healthcare policy-makers and commissioners as well as older adults themselves. The aim of this would be to shift attitudes about the inevitability of frailty and overcome some of the cultural challenges associated with niche ownership within the healthcare system, and to support the idea of integrated care for older adults. The policy-makers also recognised the need to better signpost older adult services and recommended a personal navigator to help older adults and their family caregivers to access appropriate interventions and services: this may be through the primary healthcare team, GP, a social worker or a community volunteer, but the view was that a new role, that of a well-being coordinator, was not justified.

\section{Author affiliations}

${ }^{1}$ Aston Research Centre for Healthy Ageing (ARCHA), Psychology, School of Life and Health Sciences, Aston University, Birmingham, UK

${ }^{2}$ Department of Pediatrics, Obstetrics and Gynecology, University of Valencia, Valencia, Spain

${ }^{3}$ Laboratory of Quality Assessment of Geriatric Therapies and Services, IRCCS Istituto di Ricerche Farmacologiche Mario Negri, Milan, Italy

${ }^{4}$ Family Medicine Department, Wroclaw Medical University, Wroclaw, UK ${ }^{5}$ Department of Health Research Methods, Evidence, and Impact, McMaster University, Hamilton, Canada

${ }^{6}$ Faculty of Health and Medicine, Centre for Ageing Research, Furness College, Lancaster University, Lancaster, UK

Acknowledgements The authors thank the other members of the FOCUS project for their contribution: A Nobili (IRCCS Istituto Di Ricerche Farmacologiche 'Mario Negri', Italy), A González Segura (EVERIS Spain S.L.U, Spain), A M Martinez-Arroyo (ESAM Tecnología S.L., Spain), E Bobrowicz-Campos (Nursing School of Coimbra, Portugal), F Germini (IRCCS Ca'Grande Maggiore Policlinico Hospital Foundation, Milan, Italy), J Apostolo (Nursing School of Coimbra, Portugal), L van Velsen (Roessingh Research and Development, Netherlands) and S Santana (University of Aveiro, Portugal) who were co-responsible for the design and delivery of this FOCUS work package.

Contributors $\mathrm{AC}, \mathrm{MM}$ and $\mathrm{CH}$ conceptualised the FOCUS project while $\mathrm{CH}$ conceptualised this study. All authors participated in questions design (Appendix 2). HG, E-AJD, BD, DK and TK interviewed participants, and transcribed and/or translated interviews. Participants were recruited by $\mathrm{CH}, \mathrm{BD}, \mathrm{MM}, \mathrm{DK}, \mathrm{E}-\mathrm{AJD}$ and MB-F. Analysis and interpretation of the data were conducted by HG with input from
$\mathrm{RS}$ and $\mathrm{CH}$. $\mathrm{HG}$ prepared the preliminary manuscript with all authors contributing to later drafts or critical revision of important intellectual content. $\mathrm{CH}$ and $\mathrm{AC}$ managed the study. All authors have approved this version to be published.

Funding This work was supported by the Consumers, Health, Agriculture and Food Executive Agency (CHAFEA) of the European Commission, under the European Union Health Programme (2014-2020) and for Wroclaw Medical University in Poland additionally: Ministry of Science and Higher Education in Poland (funding in years 2015-2018 allocated for the international co-financed project). The survey forms part of a larger study, 'Frailty Management Optimisation through EIP-AHA Commitments and Utilisation of Stakeholders Input' (Grant number 664367 FOCUS).

Competing interests $\mathrm{CH}$ has received research grants from ExtraCare Charitable Trust, and DK received a research grant from the Ministry of Science and Higher Education in Poland.

Ethics approval Aston University Research Ethics Committee, \#844.

Provenance and peer review Not commissioned; externally peer reviewed.

Data sharing statement This is a qualitative study and therefore the data generated are not suitable for sharing beyond that contained within the report. Further information can be obtained from the corresponding author.

Open Access This is an Open Access article distributed in accordance with the Creative Commons Attribution Non Commercial (CC BY-NC 4.0) license, which permits others to distribute, remix, adapt, build upon this work non-commercially, and license their derivative works on different terms, provided the original work is properly cited and the use is non-commercial. See: http://creativecommons.org/ licenses/by-nc/4.0/

(C) Article author(s) (or their employer(s) unless otherwise stated in the text of the article) 2018. All rights reserved. No commercial use is permitted unless otherwise expressly granted.

\section{REFERENCES}

1. Lang IA, Hubbard RE, Andrew MK, et al. Neighborhood deprivation, individual socioeconomic status, and frailty in older adults. $\mathrm{J} \mathrm{Am}$ Geriatr Soc 2009;57:1776-80.

2. Topinková E. Aging, disability and frailty. Ann Nutr Metab 2008;52:6-11.

3. Clegg A, Young J, lliffe S, et al. Frailty in elderly people. Lancet 2013;381:752-62.

4. Ferrucci L, Guralnik JM, Studenski S, et al. Designing randomized, controlled trials aimed at preventing or delaying functional decline and disability in frail, older persons: a consensus report. J Am Geriatr Soc 2004;52:625-34.

5. Gill TM, Baker DI, Gottschalk M, et al. A program to prevent functional decline in physically frail, elderly persons who live at home. N Engl J Med 2002;347:1068-74.

6. Cameron ID, Fairhall N, Langron C, et al. A multifactorial interdisciplinary intervention reduces frailty in older people: randomized trial. BMC Med 2013;11:1-10.

7. Theou O, Stathokostas L, Roland KP, et al. The effectiveness of exercise interventions for the management of frailty: a systematic review. J Aging Res 2011;2011:1-19.

8. Ng TP, Feng L, Nyunt MS, et al. Nutritional, Physical, Cognitive, and Combination Interventions and Frailty Reversal Among Older Adults: A Randomized Controlled Trial. Am J Med 2015;128:1225-36.

9. Andreasen $\mathrm{J}$, Lund $\mathrm{H}$, Aadahl $\mathrm{M}$, et al. The experience of daily life of acutely admitted frail elderly patients one week after discharge from the hospital. Int J Qual Stud Health Well-being 2015;10:27370.

10. Cesari M, Marzetti E, Thiem U, et al. The geriatric management of frailty as paradigm of "The end of the disease era". Eur J Intern Med 2016;31:11-14

11. Kapan A, Luger $E$, Haider $S$, et al. Fear of falling reduced by a lay led home-based program in frail community-dwelling older adults: A randomised controlled trial. Arch Gerontol Geriatr 2017;68:25-32.

12. Galvin KT, Todres L. Kinds of well-being: A conceptual framework that provides direction for caring. Int J Qual Stud Health Well-being 2011;6:10362.

13. Cherniack EP, Florez HJ, Troen BR. Emerging therapies to treat frailty syndrome in the elderly. Altern Med Rev 2007;12:246-58.

14. Fried LP, Tangen CM, Walston J, et al. Frailty in older adults: evidence for a phenotype. J Gerontol A Biol Sci Med Sci 2001;56:M146-57.

15. Fried LP, Ferrucci L, Darer J, et al. Untangling the concepts of disability, frailty, and comorbidity: implications for improved targeting and care. J Gerontol A Biol Sci Med Sci 2004;59:M255-63. 
16. Rockwood K, Andrew M, Mitnitski A. A comparison of two approaches to measuring frailty in elderly people. J Gerontol A Biol Sci Med Sci 2007;62:738-43.

17. Rockwood K, Mitnitski A. Frailty defined by deficit accumulation and geriatric medicine defined by frailty. Clin Geriatr Med 2011;27:17-26.

18. Walston J, Hadley EC, Ferrucci L, et al. Research agenda for frailty in older adults: toward a better understanding of physiology and etiology: summary from the American Geriatrics Society/National Institute on Aging Research Conference on Frailty in Older Adults. J Am Geriatr Soc 2006;54:991-1001.

19. Rodríguez-Mañas L, Féart C, Mann G, et al. Searching for an operational definition of frailty: a Delphi method based consensus statement: the frailty operative definition-consensus conference project. J Gerontol A Biol Sci Med Sci 2013;68:62-7.

20. Langlois F, Vu TT, Kergoat MJ, et al. The multiple dimensions of frailty: physical capacity, cognition, and quality of life. Int Psychogeriatr 2012;24:1429-36.

21. Todres L, Galvin K, Dahlberg K. Lifeworld-led healthcare: revisiting a humanising philosophy that integrates emerging trends. Med Health Care Philos 2007;10:53-63.

22. Luger E, Dorner TE, Haider S, et al. Effects of a home-based and volunteer-administered physical training, nutritional, and social support program on malnutrition and frailty in older persons: a randomized controlled trial. J Am Med Dir Assoc 2016;17:671.e9-16.

23. Cadore EL, Casas-Herrero A, Zambom-Ferraresi F, et al. Multicomponent exercises including muscle power training enhance muscle mass, power output, and functional outcomes in institutionalized frail nonagenarians. Age 2014;36:773-85.

24. Apóstolo J, Cooke R, Bobrowicz-Campos E, et al. Systematic review of effectiveness of frailty interventions with recommendations. JBI Database System Rev Implement Rep. In Press. 2017.

25. D'Avanzo B, Shaw R, Riva S, et al. Stakeholders' views and experiences of care and interventions for addressing frailty and pre-frailty: a meta-synthesis of qualitative evidence. PLoS One 2017;12:e0180127.

26. Shaw RL, Gwyther H, Holland C, et al. Understanding frailty: meanings and beliefs about screening and prevention across key stakeholder groups in Europe. Ageing Soc 2017;128:1-30.

27. Cano A, Kurpas D, Bujnowska-Fedak MM, et al. FOCUS: Frailty Management Optimisation through EIPAHA Commitments and Utilisation of Stakeholders' Input - an innovative European Project in elderly care*. Fam Med Prim Care Rev 2016;3:373-6.
28. Tong A, Sainsbury P, Craig J. Consolidated criteria for reporting qualitative research (COREQ): a 32-item checklist for interviews and focus groups. Int J Qual Health Care 2007;19:349-57.

29. Braun V, Clarke V. Using thematic analysis in psychology. Qual Res Psychol 2006;3:77-101.

30. Bodenheimer T, Wagner EH, Grumbach K. Improving primary care for patients with chronic illness. JAMA 2002;288:1775-9.

31. Wagner EH, Davis C, Schaefer J, et al. A survey of leading chronic disease management programs: are they consistent with the literature? Manag Care Q 1999;7:56-66.

32. Coleman K, Austin BT, Brach C, et al. Evidence On The Chronic Care Model In The New Millennium. Health Aff 2009;28:75-85.

33. National Institute for Health and Care Excellence. Multimorbidity: clinical assessment and management. 2016. https://www.nice.org. uk/guidance/ng56

34. Ajzen I. The theory of planned behavior. Organ Behav Hum Decis Process 1991;50:179-211.

35. Koelen MA, Lindström B. Making healthy choices easy choices: the role of empowerment. Eur J Clin Nutr 2005;59:S10-16.

36. Bandura A. Social foundations of thought and action; a social cognitive theory. NJ: Prentice Hall: Englewood Cliffs, 1986.

37. Borg Xuereb C, Shaw RL, Lane DA. Patients' and physicians' experiences of atrial fibrillation consultations and anticoagulation decision-making: A multi-perspective IPA design. Psychol Health 2016;31:436-55.

38. Shaw RL, Pattison HM, Holland C, et al. Be SMART: examining the experience of implementing the NHS Health Check in UK primary care. BMC Fam Pract 2015;16:1.

39. Clavel N, De Coster C, Pomey MP, et al. Appropriateness for Total Joint Replacement: Perspectives of Decision-Makers. Healthc Policy 2016;11:80-92.

40. Elshaug AG, Hiller JE, Moss JR. Exploring policy-makers' perspectives on disinvestment from ineffective healthcare practices. Int J Technol Assess Health Care 2008;24:1-9.

41. Guest G, Bunce A, Johnson L. How Many Interviews Are Enough? Field methods 2006;18:59-82.

42. Jette DU, Grover L, Keck CP. A qualitative study of clinical decision making in recommending discharge placement from the acute care setting. Phys Ther 2003;83:224-36.

43. Bowen GA. Naturalistic inquiry and the saturation concept: a research note. Qual Res 2008;8:137-52.

44. Morse JM. The significance of saturation. Thousand Oaks, CA: Sage Publications Sage CA. 1995. 
Correction: Understanding frailty: a qualitative study of

European healthcare policy-makers' approaches to frailty screening and management

Gwyther H, Shaw R, Jaime Dauden E, et al. Understanding frailty: a qualitative study of European healthcare policy-makers' approaches to frailty screening and management. BMJ Open 2018;8:e018653. doi: 10.1136/bmjopen-2017-018653.

At the time this research was done, author Maura Marcucci was affiliated with:

Geriatric Unit, IRCCS Ca' Granda Maggiore Policlinico Hospital Foundation, Milan, Italy; Department of Clinical Sciences \& Community Health, University of Milan, Milan, Italy

The new list of affiliations should read:

Holly Gwyther, ${ }^{1}$ Rachel Shaw, ${ }^{1}$ Eva-Amparo Jaime Dauden, ${ }^{2}$ Barbara D'Avanzo, ${ }^{3}$ Donata Kurpas, ${ }_{4}^{4}$ Maria Bujnowska-Fedak, ${ }^{4}$ Tomasz Kujawa, ${ }^{4}$ Maura Marcucci, ${ }^{5,6}$ Antonio Cano, ${ }^{2}$ Carol Holland $^{7}$

1. Aston Research Centre for Healthy Ageing (ARCHA), Psychology, School of Life and Health Sciences, Aston University, Birmingham, UK

2. Department of Pediatrics, Obstetrics and Gynecology, University of Valencia, Valencia, Spain

3. Laboratory of Quality Assessment of Geriatric Therapies and Services, IRCCS - Istituto di Ricerche Farmacologiche Mario Negri, Milan, Italy

4. Family Medicine Department, Wroclaw Medical University, Wroclaw, UK

5. 5. Geriatric Unit, IRCCS Ca' Granda Maggiore Policlinico Hospital Foundation, Milan, Italy; Department of Clinical Sciences \& Community Health, University of Milan, Milan, Italy

6. Department of Health Research Methods, Evidence, and Impact, McMaster University, Hamilton, Canada

7. Faculty of Health and Medicine, Centre for Ageing Research, Furness College, Lancaster University, Lancaster, UK

Open Access This is an Open Access article distributed in accordance with the Creative Commons Attribution Non Commercial (CC BY-NC 4.0) license, which permits others to distribute, remix, adapt, build upon this work non-commercially, and license their derivative works on different terms, provided the original work is properly cited and the use is non-commercial. See: http://creativecommons.org/licenses/by-nc/4.0/

C Article author(s) (or their employer(s) unless otherwise stated in the text of the article) 2018. All rights reserved. No commercial use is permitted unless otherwise expressly granted.

BMJ Open 2018;8:e018653corr1. doi:10.1136/bmjopen-2017-018653corr1

Check for updates 